\title{
Assessment of psychological distress in university students: A Quantitative study
}

Fradelos C. Evangelos ${ }^{1}$, Kapsiocha Eirini ${ }^{1}$, Anna Neroliatsou ${ }^{2}$, Kastanidou Sofia ${ }^{1}$, Tsaras Konstantinos $^{1}$, Papagiannis Dimitrios ${ }^{1}$, Papathanasiou V. Ioanna ${ }^{1}$ 1General Department, University of Thessaly, Larisa, Greece

2University Hospital of Larisa, Larisa, Greece

\section{Abstract.}

Introduction: Psychological health is the expression of harmonic expression of human's personality and is often disrupted by multiple factors. Especially during academic years human has to confront various stressful situations that endanger his Psychological health.

Aim: This study aims to assess psychological distress in university students and to examine possible factors that are associated with it.

Material and Methods: A cross sectional study was conducted in which 206 university students were participated. Data were collected by the Hospital Anxiety and Depression Scale, HADS and a sheet contain information regarding demographic, social and academic background special design for the purpose of the study. Spss v. 21 was used for analyzing data and the signicant level was set in $\mathrm{p} \leq 0,05$.

Results: From the total of 206 students 76,2\% were women (n=157) and 23,8\% were men with a mean age $30.1 \pm 7.15$. Anxiety symptoms were negative associated with cohabitation and marital status $(\mathrm{p}=.019$ and $\mathrm{p}=.001$ respectively). While place of residence was also negative associated $(\mathrm{p}=.000)$. While monthly income and duration of were associated with the experience of anxiety symptoms. One the other hand depression was associated with sex $(\mathrm{p}=.004)$ place of residence and origins of students ( $\mathrm{p}=.038$ and $\mathrm{p}=.058$ respectively). Finally the number of uncompleted courses and sexual preferences were associated with depression to $(\mathrm{p}=.009)$.

Conclusions: Within the context of student life that is characterized by anxiety, crises of interpersonal relationships and uncertainty maintaining student's mental health and wellbeing must be a top propriety among university services.

Keywords. Anxiety, depression, distress, University students 